\title{
Prognostic Significance of Mitochondrial Transcription Factor A Expression in Patients with Right- or Left-sided Colorectal Cancer
}

\author{
TAKEFUMI KATSUKI ${ }^{1}$, YOSHIFUMI NAKAYAMA ${ }^{1,2}$, MASAKI AKIYAMA $^{1,2}$, \\ YUSUKE SAWATSUBASHI ${ }^{1,2}$, JUN NAGATA $^{1,2}$, NORITAKA MINAGAWA ${ }^{1}$, \\ TAKAYUKI TORIGOE ${ }^{1}$, HIROTO IZUMI ${ }^{3}$, KIMITOSHI KOHONO ${ }^{4}$ and KEIJI HIRATA ${ }^{1}$ \\ ${ }^{1}$ Department of Surgery 1, School of Medicine, \\ University of Occupational and Environmental Health, Kitakyushu, Japan; \\ ${ }^{2}$ Department of Gastroenterological and General Surgery, \\ Wakamatsu Hospital of University of Occupational and Environmental Health, Kitakyushu, Japan; \\ ${ }^{3}$ Department of Occupational Pneumology, Institute of Industrial Ecological Science, \\ University of Occupational and Environmental Health, Kitakyushu, Japan; \\ ${ }^{4}$ Asahi Matsumoto Hospital, Kitakyushu, Japan
}

\begin{abstract}
Background/Aim: Mitochondrial transcription factor A (mtTFA) is necessary for both the transcription and maintenance of mitochondrial DNA (mtDNA). The present study investigated the clinical significance of mtTFA in patients with right- and left-sided colorectal cancer (CRC). Patients and Methods: Surgical specimens from 237 CRC patients were immunohistochemically stained with polyclonal anti-mtTFA antibody. The relationships among the mtTFA expression, clinicopathological factors and prognosis were evaluated. Results: Thirty-five (60.3\%) of 58 right-sided CRC patients and 82 (45.8\%) of 179 left-sided CRC patients showed high mtTFA expression. The mtTFA expression significantly correlated with lymph node metastasis, distant metastasis, the TNM stage and lymphatic invasion in leftsided CRC patients and did not correlate with any factors in right-sided CRC patients. Univariate and multivariate analyses revealed the mtTFA expression to be a significant prognostic factor in left-sided CRC patients but not in rightsided CRC patients. Conclusion: These results suggest that a high mtTFA expression is a useful marker for tumor progression and a poor prognosis in left-sided CRC patients.
\end{abstract}

Correspondence to: Yoshifumi Nakayama, Department of Surgery 1, School of Medicine, University of Occupational and Environmental Health, 1-1 Iseigaoka, Yahata-nishi-ku, Kitakyushu 807-8555, Japan. Tel: +81 936917441, Fax: +81 936032361, e-mail: nakayama@med.uoeh-u.ac.jp

Key Words: Mitochondrial transcription factor A, colorectal cancer, mitochondrial DNA.
In Japan, colorectal cancer (CRC) was estimated to be the most frequent cause of cancer-related deaths in females and the third-most frequent cause of cancer related deaths in males in 2014 (1). CRC is also one of the most lethal cancers in the world. With recent advances in chemotherapy, the overall survival of metastatic CRC has improved compared to 10 years ago. However, the prognosis of CRC still remains relatively poor. Therefore, the identification of prognostic factors that can select CRC patients at high risk for recurrence and that can predict chemosensitivity are needed.

The right-sided segment of the large bowel, including the appendix, cecum, ascending colon and transverse colon, arises from the embryonic midgut and receives blood flow from the superior mesenteric artery. In contrast, the left-sided segment of the large bowel from the splenic flexure to the upper anal canal arises from the embryonic hindgut and receives blood flow from the inferior mesenteric artery. This gives rise to differences in the clinicopathologic, cytogenetic and molecular features between right- and left-sided CRC (2). Microsatellite instability (MSI) and chromosomal instability (CIN) are the two most frequently mentioned of these genetic differences. MSI-high tumors have been reported to be more frequent in right-sided CRC (3-5), while CIN has been reported to be more frequent in the left-sided CRC (3-5). CpG island methylator phenotype (CIMP) results from hypermethylation of cytosine at the $\mathrm{CpG}$ island in the gene promoter, which leads to tumor suppressor gene silencing and carcinogenesis. CIMP was found to be significantly associated with right-sided CRC (6), and independently associated with a markedly poor prognosis in overall CRC patients (7). 
Mitochondrial transcription factor A (mtTFA) is a member of the high mobility group (HMG)-box protein family (8) and stimulates the transcription of mitochondrial genes by binding to the mitochondrial displacement loop (D-loop) region $(9,10)$. mtTFA is involved in not only the transcription of mtDNA but also in its replication, recognition of mtDNA damage, stabilization of mtDNA and, indirectly, in the repair of mtDNA (11).

Several clinical studies have examined the status of the mtTFA expression in endometrioid adenocarcinoma $(12,13)$, CRC (14) and pancreatic ductal adenocarcinoma $(15,16)$. Our previous study in a small population indicated that high mtTFA expression in CRC significantly correlated with lymph node metastasis, distant metastasis and advanced TNM staging. The survival of patients with high mtTFA expression was significantly worse than that of patients with low mtTFA expression. High mtTFA expression seems to be a useful marker for tumor progression and a poor prognosis in patients with CRC (14).

However, the significance of mtTFA expression in rightand left-sided CRC patients has not yet been evaluated. Therefore, the present study investigated the difference in the clinical significance of mtTFA expression between right- and left-sided CRC patients.

\section{Patients and Methods}

Patients. A total of 58 right-sided CRC patients and 179 left-sided CRC patients who underwent surgery at the Department of Surgery 1, University Hospital of Occupational and Environmental Health (UOEH), Japan, from 1997 to 2003 were recruited to this study. All of the intended procedures in the present study, including the use of specimens from human subjects, were approved by the Ethics Committee of UOEH. The clinical data of these patients are summarized in Table I. No patients had received chemotherapy or radiotherapy before surgery. The clinicopathological findings were determined according to the UICC tumor-node-metastasis (TNM) classifications (17).

Anti-mtTFA antibody. Regarding the immunohistochemical staining of mtTFA, the anti-mtTFA polyclonal antibody was generated by multiple immunizations of a New Zealand white rabbit using synthetic peptides, as described previously (18). This antibody has been described in previous manuscripts $(13,14,16)$.

Immunohistochemical staining of mtTFA and the evaluation of mtTFA. Immunohistochemical staining of mtTFA was performed on formalinfixed $2-\mu \mathrm{m}$ sections of tissues embedded in paraffin. These sections were deparaffinized in xylene and then rehydrated. Endogenous peroxidase was blocked with $0.3 \%$ hydrogen peroxidase in methanol for $10 \mathrm{~min}$. After washing with phosphate-buffered saline (PBS), the sections were preincubated with $10 \%$ rabbit serum albumin in PBS for $10 \mathrm{~min}$ at room temperature. The slides were then incubated with the anti-mtTFA antibody for $2 \mathrm{~h}$ at room temperature (dilution 1:400). Antibody binding was visualized using the EnVision+ Dual link system with diaminobenzidine as the chromogen (Dako Cytomation, Kyoto, Japan). The slides were counterstained with methyl green and mounted. Immunostained slides were analyzed independently by two researchers. Differences were resolved by simultaneous viewing. The expression of mtTFA in the CRC samples was evaluated according to the methods previously described $(13,14)$. The cases were judged as "(-)" or " $( \pm)$ " when no immunostaining was identified or when only minimal occasional staining $(<5 \%)$ was present, respectively, focally positive (+) when staining of at least $5 \%$ but less than $50 \%$ of the tumor cells showed immunoreactivity, and diffusely positive (++) when at least $50 \%$ of the tumor cells showed immunoreactivity. Finally, the cases were classified into two groups based on low (- or $\pm)$ or high $(+$ or ++$)$ expression.

Immunohistochemical staining of ssDNA and the evaluation of the apoptotic Index. Immunohistochemical staining of single-stranded DNA (ssDNA) was performed on formalin-fixed 2- $\mu$ m sections of tissues embedded in paraffin. These sections were deparaffinized in xylene and then rehydrated. After endogenous peroxidase was blocked, the sections were preincubated with $10 \%$ goat serum albumin in PBS for $15 \mathrm{~min}$ at room temperature. The slides were then incubated with the ssDNA antibody (IBL, Gunma Japan) for $60 \mathrm{~min}$ at a room temperature (dilution, 1:100). Antibody binding was visualized using the EnVision+ Rabbit/IIRP (Dako Cytomation) with diaminobenzidine as the chromogen. The slides were counterstained with Mayer hematoxylin and mounted. ssDNA staining was evaluated in each section by counting the frequency of labeled cells in 5 high-power fields containing 100 tumor cells each. The apoptotic index (AI) was defined as the percentage of tumor cells with nuclei immunoreactivity among the total tumor cells.

Immunohistochemical staining of Ki-67 and the evaluation of the MIB-1 index. Immunohistochemical staining of Ki-67 was performed on formalin-fixed 2- $\mu \mathrm{m}$ sections of tissues embedded in paraffin. These sections were deparaffinized in xylene and then rehydrated. After endogenous peroxidase was blocked, the slides were subjected to microwave treatment in $10 \mathrm{mM}$ citrate buffer $(\mathrm{pH} 6.0)$ for $10 \mathrm{~min}$. After washing with PBS, the sections were preincubated with $10 \%$ rabbit serum albumin in PBS for $10 \mathrm{~min}$ at room temperature. Immunohistochemical staining for $\mathrm{Ki}-67$ antigen was performed using a monoclonal mouse anti-human Ki-67 antibody Clone MIB-1 (Dako Japan). The slides were then incubated with Ki-67 antibody for $1 \mathrm{~h}$ at room temperature (dilution 1:50). After washing with PBS, the slides were treated with anti-mouse immunoglobulin for $20 \mathrm{~min}$ and were then incubated with streptavidin-biotinylated horseradish peroxidase complex (LSAB kit/HRP: Nichirei, Tokyo, Japan) for 10 min. The slides were incubated in PBS containing diaminobenzidine and $1 \%$ hydrogen peroxidase for $10 \mathrm{~min}$, counterstained with Mayer's hematoxylin, and mounted (19). The proliferative activity of the tumor cells was assessed by the MIB-1 index, which was determined as the percentage of tumor cells showing positive staining of nuclei reactive among the total tumor cells counted (19).

Clinicopathological assessment. The tumors were staged by two pathologists who had no prior knowledge of the results of the assays, according to the UICC tumor-node-metastasis (TNM) classifications 7th edition (17). Clinicopathological factors such as age, gender, tumor size, histological type, depth of invasion, lymph node metastasis, distant metastasis and staging were analyzed for their association with the mtTFA expression.

Statistical analyses. The relationships between the parameters were also assessed statistically using the $\mathrm{c}^{2}$ test with the Stat View-J 


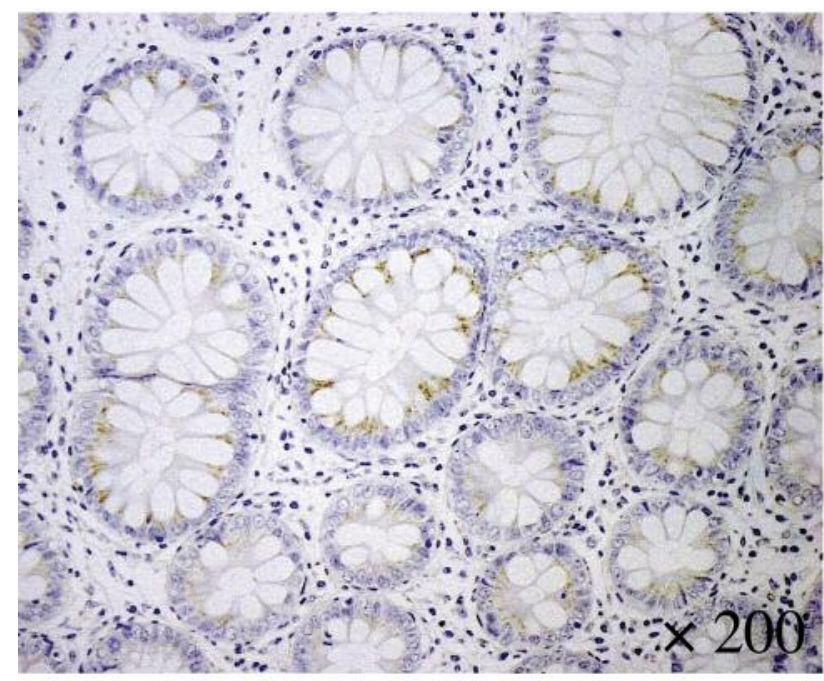

Figure 1. Immunohistochemical staining of mtTFA in normal colonic muсоsa $(\times 400)$.

software package (version 5.0, SAS institute, Inc., Cary, NC, USA). The Kaplan-Meier method was applied to determine the survival, and statistical significance was calculated using the log-rank test. Univariate and multivariate analyses of the survival were performed using the Cox proportional hazards model. Statistical significance was established at the $p \leq 0.05$ level.

\section{Results}

Table I shows the profiles of the 237 patients diagnosed with primary right-sided (58) and left-sided (179) CRC recruited for the present study. Immunohistochemical staining of endogenous mtTFA was performed on 58 right-
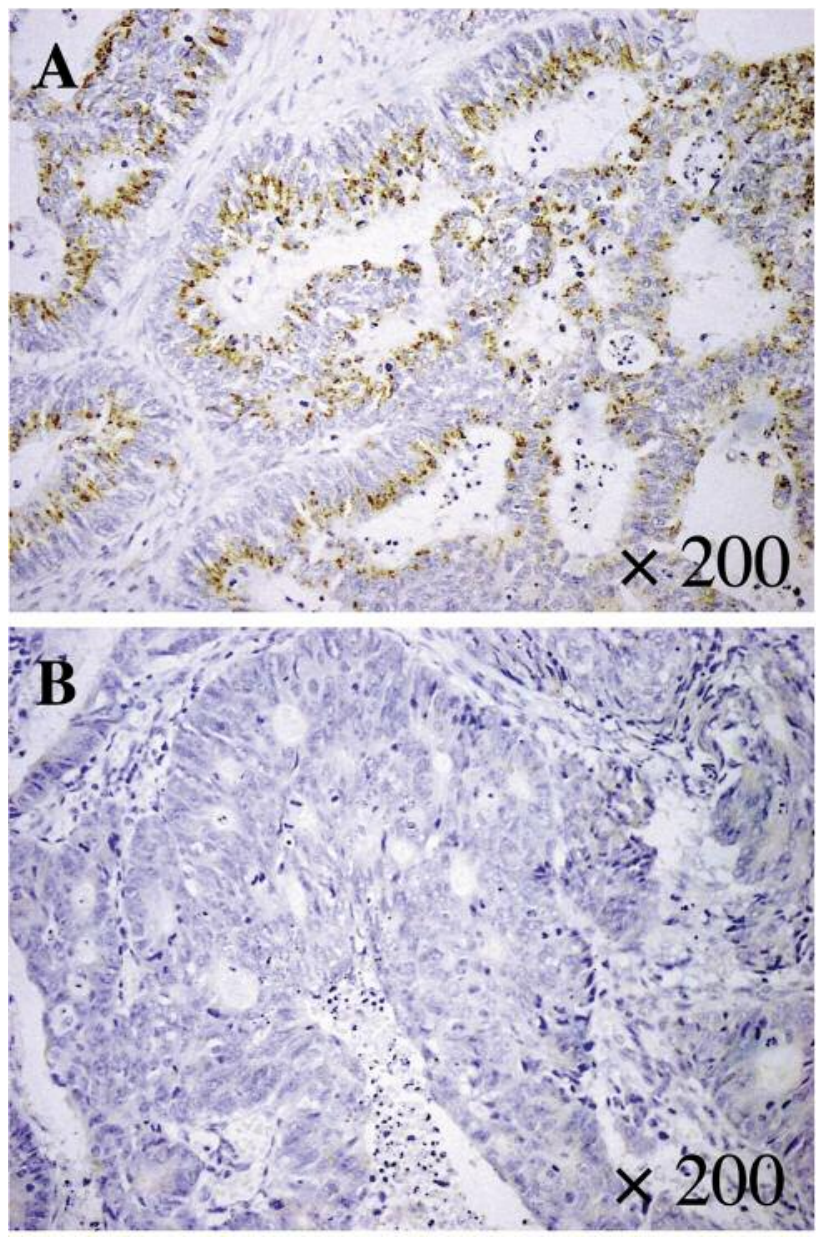

Figure 2. Immunohistochemical staining of mtTFA in colorectal cancer specimens. Positive expression (++) of mtTFA (A) and negative expression (-) of mtTFA $(B)(\times 400)$.
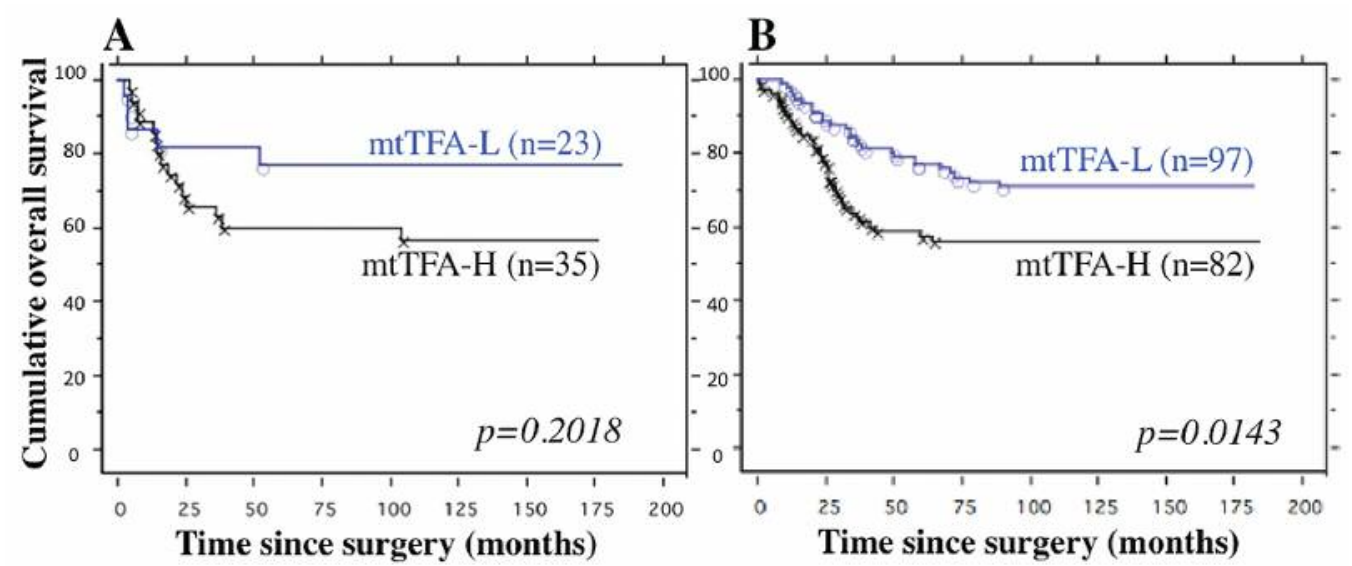

Figure 3. The prognostic significance of mtTFA expression was analyzed using the Kaplan-Meier method in the patients with right-sided CRC ( $n=58$ ) (A) and left-sided CRC (n=179) (B). The patients were divided into low and high groups according to the previous classification (14). The patients in the low mtTFA (mtTFA-L) group showed a significantly better prognosis in comparison to those in the high mtTFA (mtTFA-H) group in the patients with left-sided CRC ( $p=0.0143)(B)$ but not in the patients with right-sided CRC (( $p=0.2018)(A)$. 
Table I. Characteristics of the patients with right-sided or left-sided colorectal cancer.

\begin{tabular}{lccc}
\hline & Right-sided & Left-sided & $p$-Value \\
\hline No. of patients & 58 & 179 & \\
Site C/A/T & $6 / 34 / 18$ & & \\
$\quad$ D/S/Rs/Ra/Rb & \multicolumn{2}{c}{$13 / 53 / 41 / 36 / 36$} \\
Gender (M/F) & $28 / 30$ & $103 / 76$ & 0.2174 \\
Age (yr) (mean \pm S.D.) & $65.5 \pm 12.1$ & $65.5 \pm 10.8$ & 0.9934 \\
Tumor size (cm) (mean \pm S.D.) & $5.6 \pm 2.7$ & $5.1 \pm 1.8$ & 0.1046 \\
Depth of invasion & & & \\
Tis/T1/T2/T3/T4a/T4b & $1 / 1 / 5 / 37 /$ & $1 / 2 / 28 /$ & 0.5853 \\
& $11 / 3$ & $95 / 42 / 11$ & \\
Lymph node metastasis (-/+) & $31 / 27$ & $85 / 94$ & 0.4299 \\
Distant metastasis (-/+) & $43 / 15$ & $148 / 31$ & 0.1528 \\
TNM stage & & & \\
0/I/II/IIIA/IIIB/IIIC/IV & $1 / 4 / 24 / 2 /$ & $1 / 22 / 58 /$ & 0.1883 \\
& $7 / 5 / 15$ & $6 / 48 / 13 / 31$ & \\
Histological type & & & \\
$\quad$ Differentiated & 49 & 169 & 0.0155 \\
$\quad$ Undifferentiated & 9 & 10 & \\
Lymphatic invasion (Weak/Strong) & $30 / 28$ & $91 / 88$ & 0.9066 \\
Venous invasion (-/+) & $30 / 28$ & $83 / 96$ & 0.4779 \\
\hline
\end{tabular}

The weak group of lymphatic invasion indicated score 0 and 1 , and the strong group indicated score 2 and 3 by the Japan Classification of Colorectal Cancer.

sided and 179 left-sided CRC specimens. The expression of mtTFA was observed in the cytoplasm of the normal colonic mucosa (Figure 1). Positive signals (++) for mtTFA were observed in the cytoplasm of cancer cells (Figure 2A). Negative signals (-) for mtTFA were observed in the cytoplasm of cancer cells (Figure 2B). Thirty-five (60.3\%) of the 58 right-sided CRC patients and $82(45.8 \%)$ of the 179 left-sided CRC patients had high mtTFA expression.

The patients were divided into high- and low-MI groups, with the mean value of $43.6 \%$ as the cut-off value. A total of 125 patients (27 right-sided CRCs, 98 left-sided CRCs) had a high-MI, and 112 (31 right-sided CRCs, 81 left-sided CRCs) had a low-MI. The patients were also divided into high- and low-AI groups, with the mean value of $5.1 \%$ as the cut-off value. A total 124 patients (40 right-sided CRCs, 84 left-sided CRCs) had a high-AI, and 113 patients (18 right-sided CRCs, 95 left-sided CRCs) had a low-AI.

Based on the evaluation of the mtTFA immunostaining, the mtTFA expression significantly correlated with lymph node metastasis, distant metastasis, TNM stage and lymphatic invasion in left-sided CRC patients (Table II). In contrast, the mtTFA expression did not correlate with any factors in right-sided CRC patients (Table II).

Kaplan-Meier analyses for the overall survival based on the mtTFA expression were also performed (Figure 3 ). The median follow-up time was 93.06 months (range $=1.0-185$ months). The
Table II. Association between mtTFA expression and clinicopathological factors, A. I. and M. I. in the patients with right- or left-sided colorectal cancer.

\begin{tabular}{|c|c|c|c|c|c|c|}
\hline & \multicolumn{3}{|c|}{$\begin{array}{l}\text { Right-sided CRC } \\
\text { mtTFA expression }\end{array}$} & \multicolumn{3}{|c|}{$\begin{array}{l}\text { Left-sided CRC } \\
\text { mtTFA expression }\end{array}$} \\
\hline & High & Low & $p$-Value & High & Low & $p$-Value \\
\hline \multicolumn{7}{|l|}{ Age } \\
\hline Young & 17 & 7 & 0.1701 & 36 & 45 & 0.7288 \\
\hline Old & 18 & 16 & & 46 & 52 & \\
\hline \multicolumn{7}{|l|}{ Gender } \\
\hline Male & 17 & 11 & 0.9557 & 45 & 58 & 0.5074 \\
\hline Female & 18 & 12 & & 37 & 39 & \\
\hline \multicolumn{7}{|l|}{ Size } \\
\hline Small & 18 & 16 & 0.1701 & 51 & 59 & 0.5074 \\
\hline Big & 17 & 7 & & 31 & 38 & \\
\hline \multicolumn{7}{|c|}{$\mathrm{T}$} \\
\hline$\leq \mathrm{T} 3$ & 25 & 19 & 0.3304 & 52 & 74 & 0.0601 \\
\hline $\mathrm{T} 4$ & 10 & 4 & & 30 & 23 & \\
\hline \multicolumn{7}{|l|}{$\mathrm{N}$} \\
\hline$(-)$ & 16 & 15 & 0.1452 & 32 & 53 & 0.0371 \\
\hline$(+)$ & 19 & 8 & & 50 & 44 & \\
\hline \multicolumn{7}{|l|}{$\mathrm{M}$} \\
\hline$(-)$ & 25 & 18 & 0.5610 & 60 & 88 & 0.0020 \\
\hline$(+)$ & 10 & 5 & & 22 & 9 & \\
\hline \multicolumn{7}{|l|}{ Stage } \\
\hline$\leq \mathrm{IIIB}$ & 20 & 18 & 0.0979 & 54 & 81 & 0.0063 \\
\hline IIIC $\leq$ & 15 & 5 & & 28 & 16 & \\
\hline \multicolumn{7}{|l|}{$\mathrm{Hx}$} \\
\hline Differentiated & 28 & 21 & 0.2448 & 78 & 91 & 0.7043 \\
\hline Undifferentiated & 7 & 2 & & 4 & 6 & \\
\hline \multicolumn{7}{|l|}{ ly } \\
\hline Weak & 18 & 12 & 0.9557 & 35 & 56 & 0.0448 \\
\hline Strong & 17 & 11 & & 47 & 41 & \\
\hline \multicolumn{7}{|c|}{$\mathrm{v}$} \\
\hline$(-)$ & 18 & 12 & 0.9557 & 38 & 45 & 0.9946 \\
\hline$(+)$ & 17 & 11 & & 44 & 52 & \\
\hline \multicolumn{7}{|l|}{ A.I. } \\
\hline Low & 14 & 4 & 0.0687 & 42 & 53 & 0.6478 \\
\hline High & 21 & 19 & & 40 & 44 & \\
\hline \multicolumn{7}{|l|}{ M.I. } \\
\hline Low & 18 & 13 & 0.7037 & 35 & 46 & 0.5256 \\
\hline High & 17 & 10 & & 47 & 51 & \\
\hline
\end{tabular}

T: Depth of invasion; N: lymph node metastasis; M: distant metastasis; ly: lymphatic invasion; v: venous invasion; A.I.: apoptotic index; M.I.: MIB-1 index. The patients were divided into two groups assessed with the mean value of age or size as the cut-off value. The weak group of ly indicated score 0 and 1 , and the strong group indicated score 2 and 3 by the Japan Classification of Colorectal Cancer.

survival of patients with low mtTFA expression was not significantly better than that of those with high mtTFA expression $(p=0.2018$, Figure 3A) in right-sided CRC patients. However, the survival of patients with low mtTFA expression was significantly better than that of those with high mtTFA expression ( $p=0.0143$, Figure 3B) in left-sided CRC patients. 
Table III. Univariate analysis for clinicopathological factors, apoptotic index, MIB-1 index and the expression of mtTFA in the patients with right-sided or left-sided colorectal cancer.

\begin{tabular}{|c|c|c|}
\hline \multirow[t]{2}{*}{ Factor } & \multicolumn{2}{|c|}{$\begin{array}{c}p \text {-Value of } \\
\text { univariate analysis }\end{array}$} \\
\hline & Right-sided & Left-sided \\
\hline TNM stage (I, II, IIIA, IIIB vs. IIIC, IV) & $<0.0001$ & $<0.0001$ \\
\hline $\mathrm{T}(\mathrm{T} 1, \mathrm{~T} 2, \mathrm{~T} 3$ vs. T4) & $<0.0001$ & $<0.0001$ \\
\hline $\mathrm{N}(-v s .+)$ & $<0.0001$ & $<0.0001$ \\
\hline$M(-v s .+)$ & $<0.0001$ & $<0.0001$ \\
\hline Lymphatic invasion (Weak vs. Strong) & 0.0005 & $<0.0001$ \\
\hline Venous invasion $(-v s .+)$ & 0.0665 & $<0.0001$ \\
\hline Histological type (Diff. vs. Undiff.) & 0.0464 & $<0.0001$ \\
\hline MIB-1 index (Low vs. High) & 0.9141 & $<0.0001$ \\
\hline Apoptotic index (Low $v s$. High) & 0.2093 & 0.0009 \\
\hline Size (Small vs. Big) & 0.3738 & 0.0015 \\
\hline mtTFA expression (Low vs. High) & 0.2018 & 0.0143 \\
\hline Gender (M vs. F) & 0.2840 & 0.1966 \\
\hline Age (Young $v s$. oOd) & 0.8034 & 0.1898 \\
\hline
\end{tabular}

The patients were divided into young and old groups assessed with the mean value of age as the cut-off value, and into small and big groups assessed with the mean value in diameter as the cut-off value. The weak group of ly indicated score 0 and 1 , and the strong group indicated score 2 and 3 by the Japan Classification of Colorectal Cancer.

In right-sided CRC patients, a univariate analysis indicated that the TNM stage, depth of invasion, lymph node metastasis, distant metastasis, lymphatic invasion and histological type were significant prognostic factors (Table III), and a multivariate analysis indicated that the depth of invasion, lymph node metastasis and distant metastasis were significant independent prognostic factors (Table IV). In contrast, in left-sided CRC patients, a univariate analysis indicated that the TNM stage, depth of invasion, lymph node metastasis, distant metastasis, lymphatic invasion, vessel invasion, histological type, MIB-1 index, apoptotic index, tumor size and the mtTFA expression were significant prognostic factors (Table III) and a multivariate analysis indicated that lymph node metastasis, distant metastasis, vessel invasion, MIB-1 index and the mtTFA expression were significant independent prognostic factors (Table IV).

\section{Discussion}

The findings regarding the prognosis of right-sided versus left-sided colorectal cancer are conflicting at present. Some studies have revealed a poor survival with a right-sided primary tumor location (20-24), while others have revealed poor or a similar survival based on a left-sided primary tumor location $(25,26)$. As the criteria for the recruitment of patients differ among these previous manuscripts, it may be
Table IV. Multivariate analysis for clinicopathological factors, apoptotic index, MIB-1 index and the expression of mtTFA in the patients with right-sided or left-sided colorectal cancer.

\begin{tabular}{lcc}
\hline Factor & \multicolumn{2}{c}{$\begin{array}{c}p \text {-Value of } \\
\text { multivariate analysis }\end{array}$} \\
\cline { 2 - 3 } & Right sided & Left sided \\
\hline TNM stage (I-IIIB $v s$. IIIC, IV) & 0.0731 & 0.1092 \\
T (T1, T2, T3 vs. T4a, T4b) & 0.0363 & 0.7195 \\
N (- vs. +) & 0.0184 & 0.0490 \\
M (- vs. +) & 0.0105 & 0.0011 \\
Lymphatic invasion (weak $v s$. strong) & 0.8467 & 0.3270 \\
Venous invasion (- vs. +) & & 0.0141 \\
mtTFA expression (low $v s$. high) & & 0.0428 \\
Histological type (Diff. $v s$. Undiff.) & 0.1220 & 0.0748 \\
MIB-1 index (low $v s$. high) & & 0.0089 \\
Apoptotic index (high $v s$. low) & & 0.0863 \\
Size (small $v s$. large) & & 0.8264 \\
\hline
\end{tabular}

The patients were divided into small and large groups assessed with $\mathrm{t}$ the mean value of $5.6 \mathrm{~cm}$ in diameter of right sided group and $5.1 \mathrm{~cm}$ in diameter of left sided group as the cut-off value. The weak group of lymphatic invasion indicated score 0 and 1 , and the strong group indicated score 2 and 3 by the Japan Classification of Colorectal Cancer.

difficult to evaluate and compare the results of these reports. While some studies evaluated the patients with Stage I-III disease $(25,26)$, other studies evaluated those with Stage IIV $(20,21,23)$. Furthermore, some studies have evaluated patients with colon cancer $(20,21,25)$, while others have evaluated those with colorectal cancer $(26,27)$. In our study, there was no significant differences in the survival between right- and left-sided CRC patients $(p=0.7504)$ including Stage I-IV disease. It is important to clarify the differences in prognostic factors or factors predictive of the therapeutic effect based on the location of CRC.

Several reports have shown that a high mtTFA expression correlates with a worse prognosis by affecting tumor progression. In patients with endometrioid adenocarcinomas, the mtTFA expression was found to be significantly associated with the surgical stage, myometrial invasion, lymphovascular space invasion, cervical invasion and lymph node metastasis (13). In addition, this previous study indicated that the 10 -year overall survival rate of the endometrioid adenocarcinoma patients with a high mtTFA expression was significantly worse than that of patients with a low mtTFA expression by a univariate, but not a multivariate, survival analysis (13). In pancreatic ductal adenocarcinoma, Yamauchi et al. indicated that the mtTFA expression played a pivotal role in worsening the postoperative clinical course through the induction of antiapoptotic effects in pancreatic ductal adenocarcinoma cells (15). Other reports have indicated that the combination of 
mtTFA and survivin expression is an independent, novel and powerful marker for a poor prognosis in pancreatic ductal adenocarcinoma patients after surgery (16). Our previous small-scale study indicated that the survival of CRC patients with a high mtTFA expression was significantly worse than that of patients with a low mtTFA expression (14). In the present study, our data suggested that a high mtTFA expression might be a useful marker for tumor progression and a poor prognosis in left-sided CRC patients, but not in right-sided CRC patients.

Some studies have described the regulatory mechanism for the expression of mtTFA (28-33). An in vivo genomic footprinting study suggested that NRF-2 and Sp1 are probably involved in the regulation of the ITTFA gene in rat hepatoma (28). The in vitro methylation of the NRF-1 binding site suppressed the promoter activity of mtTFA (29). The overexpression of PGC-1 related coactivator (PRC) in oncocytic tumors induced mitochondrial biogenesis through NRF-1, resulting in an increase in mtTFA and mtDNA transcript levels (30). Another study described the redox regulation of NRF-1 phosphorylation and nuclear translocation by phosphatidylinositol 3,4,5-triphosphate kinase/AKt signaling, which controls the mtTFA induction by an anti-oxidant pro-survival network (31). Also, arsenic causes several human cancers. Chronic exposure to arsenic causes the increased cell survival, DNA damage and increased expression of mtTFA in human prostate epithelial cells (32). The expression of cytochrome $c$ oxygen, mtTFA, NRF-1 and PGC-1apfha was increased in arsenic-induced Bowen's disease (33). The regulatory mechanism for mtTFA is not fully understood. However, the regulation of the mtTFA expression may be important as CRC patients with a low mtTFA expression have a favorable prognosis. More extensive studies about the regulation of mtTFA are needed.

Kurita et al. indicated that cellular expression of the antiapoptotic factor BCL2L1 was down-regulated following the transfection of siRNA knockdown of mtTFA in ovarian cancer cell lines (34). In addition, $B C L 2 L 1$ promoter activity was increased after the transfection of a mtTFA expression plasmid but decreased after siRNA knockdown of mtTFA (34). mtTFA also regulates the BCL2L1 gene expression in ovarian cancer. Furthermore, increased apoptosis is observed in mtTFA-knockout mice, suggesting that mtTFA plays an important role in apoptosis (35). These studies suggested that mtTFA might be a molecular target of cancer therapy. The strong expression of mtTFA in patients with metastatic CRC was detected in 8 of 33 complete response/partial response patients $(24.2 \%)$ and 18 of 26 stable disease/progressive disease patients (69.2\%), indicating that mtTFA expression was significantly correlated with the response to chemotherapy $(p<0.01)(36)$. The median overall survival of metastatic CRCs was significantly longer in patients without mtTFA expression than in those with it (36). This report suggested that the immunohistochemical study of mtTFA may be useful for predicting the clinical outcome of metastatic CRC patients treated with the FOLFOX regimen.

In conclusion, we found that a high mtTFA expression in tumor specimens may be a useful marker for both the progression of the tumors and for a poor prognosis of patients with left-sided CRC.

\section{Conflicts of Interest}

The Authors declare that there are no conflicts of interest regarding the publication of this paper.

\section{Acknowledgements}

The Authors would like to thank Ms. Yuko Ueda for her technical assistance. This study was supported in part by a Grant-in-Aid for Scientific Research from the Ministry of Education, Science and Culture of Japan.

\section{References}

1 Center for cancer control and information services, National cancer center, Japan: Vital statistics Japan (Ministry of Health, Labour and Welfare), 2016.

2 Bufill JA: Colorectal cancer. evidence for distinct genetic categories based on proximal and distal tumor location. Ann Intern Med 113: 779-788, 1990.

3 Markowitz SD and Bertagnolli MM: Molecular origins of cancers: molecular basis of colorectal cancer. N Eng J Med 361: 2449-2460, 2009.

4 Pritchard CC and Grady WM: Colorectal cancer molecular biology moves into clinical practice. Gut 60: 116-129, 2011.

5 Gervaz P, Bucher P and Morel P: Two colons- two cancers: paradigm shift and clinical implications. J Surg Oncol 88: 261266, 2004.

6 Barault L, Charon-Barra C, Jooste V, Funes de la Vega M, Martin L, Roignot P, Rat P, Bouvier AM, Laurent-Puig P, Faivre J, Chapusot C and Piaed F: Hypermethylator phenotype in sporadic colon cancer: study on a population-based series of 582 cases. Cancer Res 68: 8541-8546, 2008.

7 Juo YY, Johnston FM, Zhang DY, Juo HH, Wang H, Pappou EP, Yu T, Easwaran H, Baylin S, van Engeland M and Ahuja N: Prognostic value of $\mathrm{CpG}$ island methylatior phenotype among colorectal cancer patients: a systemic review and meta-analysis. Ann Oncol 25: 2314-2327, 2014.

8 Parisi MA and Clayton DA: Similarity of human mitochondrial transcription factor 1 to high mobility group proteins. Science 252: 965-969, 1991.

9 Larsson NG, Wanf J, Wilhelmsson H, Oldfors A, Rustin P, Lewandoski M, Barsh $\mathrm{G}$ and Clayton DA: Mitochondrial transcription factor A is necessary for mtDNA maintenance and embryogenesis in mice. Nat Genet 18: 231-236, 1998.

10 Wallberg $M$ and Clayon DA: Sequence and properties of the human KB cell and mouseL cell D-loop regions of mitochondrial DNA. Nucleic Acid Res 9: 5411-5421, 1981. 
11 Gaspari M, Lasson NG and Gustafsson CM: The transcriptional machinery in mammalian mitochondria. Biochem Biophys Acta 1659: 148-152, 2004.

12 Cormio A, Cuerra F, Cormio G, Pesce V, Fracasso F, Loizzi V, Cantatore P, Selvaggi L and Gadaleta MN: The PGC-1 adependent pathway of mitochondrial biogenesisi is upregulated in type I endometrial cancer. Biochem Biophys Res Commun 390: 1182-1185, 2009.

13 Toki N, Kagami S, Kurita T, Kawagoe T, Matsuura Y, Hachisuga $\mathrm{T}$, Matsuyama $\mathrm{A}$, Hashimoto $\mathrm{H}$, Izumi $\mathrm{H}$ and Kohno $\mathrm{K}$ : Expression of mitochondrial transcription factor $\mathrm{A}$ in endometrial carcinomas: clinicopathologic correlations and prognostic significance. Virchows Arch 456: 387-393, 2010.

14 Nakayama Y, Yamauchi M, Minagawa N, Tarigoe T, Izumi H, Kohno $\mathrm{K}$ and Yamaguchi $\mathrm{K}$ : Clinical significance of mitochondrial transcription fantor A expression in patients with colorectal cancer. Oncol Rep 27: 1325-1330, 2012.

15 Yamauchi M, Nakayama Y, Minagawa N, Torigoe T, Shibao K and Yamaguchi K: Mitochondrial transcription factor A worsen the clinical course of patients with pancreatic cancer through inhibition of apoptosis of cancer cells. Pancreas 43: 405-410, 2014.

16 Kimura T, Kitada S, Uramoto H, Zhi L, Kawatsu Y, Takeda T, Horiw S, Nabeshima A, Nogichi H, Sasaguri Y, Izumi H, Kohno $\mathrm{K}$ and Yamada $\mathrm{S}$ : The combination of strong immunohistochemical mtTFA expression and a high surviving index predicts a shorter disease-specific survival in pancreatic ductal adenocarcinoma. Histol Histopathol 30: 193-204, 2015.

17 Sobin LH, Gospodarowicz MK, Wittekind C and editors: International Union Against Cancer (UICC) TNM classification of malignant tumors, 7th edition. New York, Wiley-Liss, 2010.

18 Yoshida Y, Izumi H, Torigoe T, Ishiguchi H, Itoh H, Kang D and Kohno K: p53 physically interacts with mitochondrial transcription factor A and differentially regulates binding to damages DNA. Cancer Res 63: 3729-3734, 2003.

19 Hasebe T, Sasaki S, Sugitoh M, Ono M, Saitoh N and Ochiai A: Proliferative activities of tumor stromal cells play important roles in tumor thicjness and progression of T3 ulcerative-type colorectal cancer. Virchows Arch 442: 569-576, 2003.

20 Benedix F, Kube R, Meyer F, Schmidt U, Gastinger I and Lippeert H: Colon/Rectum Carcinomas (Primary Tumor) Study Group. Comparison of 17,641 patients with right- and left-sided colon cancer: differences in epidermology, perioperative course, histology, and survival. Dis Colon Rectum 53: 57-64, 2010.

21 Meguid RA, Slidell MS, Wolfgang CL, Chang DC and Ahuja N: Is there a difference in survival between right- versus left-sided colon cancers? Ann Surg Oncol 15: 2388-2394, 2008.

22 Suttie SA, Shaikh I, Mullen R, Amin AI, Daniel T and Yalamarthi S: Outcome of right- and left-sided colonic and rectal cancer following surgical resection. Colorectal Dis 13: 884-889, 2011.

23 Derwinger K and Gustavsson B: Variations in demography and prognosis by colon cancer location. Anticancer Res 31: $2347-$ 2350, 2011.

24 Jess P, Hansen IO, Gamborg M and Jess T, on behalf of the Danish Colorectal Cancer Group: A nationwide Danish cohort study challenging the categorization into right-sided and leftsided colon cancer. BMJ Open 3: e002608, 2006.
25 Warschkow R, Sulz MC, Tarantino I, Schmied BM, Cerny T and Güller U: Better survival in right-sided versus left-sided stage IIII colon cancer patients. BMC Cancer 16: 554, 2016.

26 Weiss JM, Pfau PR, O'Conner ES, King J, LoConte N, Kennrdy $\mathrm{G}$ and Smith MA: Mortality by stage for for right- versus leftsided colon cancer: analysis of surveillance, epidemiology, and results-medicare data. J Clin Oncol 29: 4401-4409, 2011.

27 Wang F, Bai L, Liu TS, Yu YY, He MM, Liu KY, Luo HY, Zhang DS, Jin Y, Wang FH, Wang ZQ, Wang DS, Qin MZ, Ren $\mathrm{C}$, Li YH and Xu RH: Right- and left-sided colorectal cancers respond differently to cetuximab. Chinese J of Cancer 34: 24, 2015.

28 Dang X, Ghoshal K, Majumder S, Yadav SP and Jacob T: Mitochondrial transcriptional factor $\mathrm{A}$ and its downdtream targets are up-regulated in a rat hepatoma. J Biol Chem 277: 43309-43318, 2002.

29 Choi YS, Kim S, Lee HK, Lee KU and Pak K: On vitro methylation of nuclear respirayory factor-1 binding site suppresses the prompter activity of mitochondrial transcription factor A. Biochem Biophys Res Commun 314: 118-122, 2004.

30 Savagner F, Mirebeau D, Jacques C, Guyetant S, Morgan C, Franc $\mathrm{B}$, Reynier $\mathrm{P}$ and Malithièry $\mathrm{Y}$ : PGC-1-related coactivator and targets are upregulated in thyroid oncocytoma. Biochem Biophys Res Commun 310: 779-784, 2003.

31 Piantadosi C and Suliman HB: Mitochondrial transcription factor A induction by redox activation of nuclear respiratory factor 1 . J Biol Chem 281: 324-333, 2006.

32 Singh KP, Kumari R, Treas J and DuMond JW: Chronic exposure to arsenic causes increased cell survival, DNA damage, and increased expression of mitochondrial transcription factor $\mathrm{A}$ (mtTFA) in human prostate epithelial cells. Chem Res Toxicol 24: 340-349, 2011.

33 Lee $\mathrm{CH}$, Wu SB, Hong CH, Liao WT, Wu CY, Chen GS, Wei $\mathrm{YH}$ and $\mathrm{Yu}$ HS: Aberrant cell proliferation by enhanced mitochondrial biogenesis via mtTFA in arsenical skin cancer. Am J Path 178: 2066-2076, 2011.

34 Kurita T: Mitochondrial transcription factor A regulates BCL2L1 gene expression and is a prognostic faxtor in serous ovarian cancer. Cancer Sci 103: 239-244, 2012.

35 Wang J, Silva JP, Gustafsson CM, Rustin P and Larsson NG: Increased in vivo apoptosis in cells lacking mitochondrial DNA gene expression. Proc Natl Acad Sci USA 98: 4038-4043, 2001.

36 Yoshida Y, Hasegawa J, Nezu R, Kim YK, Hirota M, Kawano $\mathrm{K}$, Izumi $\mathrm{H}$ and Kohno K: Clinical usefulness of mitochondrial transcription factor $\mathrm{A}$ expression as a predictive marker in colorectal cancer patients treated with FOLFOX. Cancer Sci 102: 578-582, 2011.
Received September 29, 2017

Revised October 17, 2017

Accepted October 18, 2017 